\title{
ANALYTIC DISCRETE-ORDINATES SOLUTION FOR TIME-DEPENDENT TRANSPORT IN FINITE MEDIA
}

\author{
Jeffery D. Densmore and Gabriel Kooreman \\ Naval Nuclear Laboratory \\ P.O. Box 79, West Mifflin, PA 15122, USA \\ jeffery.densmore@unnpp.gov, gabriel.kooreman@unnpp.gov
}

\begin{abstract}
We present an extension of the Analytic Discrete-Ordinates method to time-dependent transport in finite media. The application of this technique to time-dependent transport is primarily accomplished through the use of a Laplace transform. In the case of finite media, a system of equations arises from enforcing boundary conditions. Instead of directly solving this system, we construct a solution in terms of a Neumann series. We then show that terms can be neglected when numerically evaluating the inverse Laplace transform such that the series reduces to a finite sum. With this extension, we use convergence acceleration to generate a high-precision benchmark.
\end{abstract}

KEYWORDS: Analytic Discrete-Ordinates Method, Time-Dependent Transport, Laplace Transform, Neumann Series, Talbot's Method, Wynn-Epsilon Convergence Acceleration

\section{INTRODUCTION}

Analytically solving the discrete-ordinates equations - that is, approximating the angular dependence using quadrature then solving the resulting space-dependent system of equations analytically - is a well-known technique for generating solutions to radiation-transport problems [1]. In particular, Barichello, Siewert, and others have cast this approach as the Analytic Discrete-Ordinates (ADO) method [2,3]. Ganapol has also used this technique along with convergence acceleration with respect to the number of directions in the quadrature set to produce high-precision benchmarks [4].

Recently, we have extended the ADO method to time-dependent transport, specifically for the case of semi-infinite media [5]. This extension was primarily accomplished through the use of a Laplace transform in time. We also investigated using convergence acceleration to generate a high-precision benchmark in the presence of ray effects, which are inherent in solutions of the discrete-ordinates equations when time dependence is included. We found that, with a properly chosen sequence of quadrature sets, we can indeed use convergence acceleration with the ADO method to produce a high-precision benchmark for time-dependent transport, too.

In this paper, we continue our extension of the ADO method to time-dependent transport to include finite media. We develop this extension in the context of solving an example problem. A new aspect in this case is a system of equations that arises from enforcing boundary conditions. Instead 
of directly solving this system, we construct a solution in terms of a Neumann series. Not only is this series solution easier to develop than a direct solution, we show that terms can be neglected when numerically evaluating the inverse Laplace transform such that the series reduces to a finite sum. We also again use convergence acceleration to generate a high-precision benchmark.

Our motivation is the lack of benchmarks for time-dependent transport in finite media. We do note that Aplin and Henderson have developed an analytic (i.e., in which the angular dependence is not approximated) benchmark for such a problem using the multiple-collision technique [6]. An ADO-based approach may have certain advantages over the multiple-collision technique, for example, it may be more easily extended to heterogeneous media (see Ref. [4] for an application of an ADO-like method to heterogeneous media). At the very least, our current extension of the ADO method to time-dependent transport in finite media complements this previous work.

\section{PROBLEM DESCRIPTION}

We specifically consider monoenergetic, planar-symmetric radiation transport in a homogeneous, isotropically scattering medium. The corresponding transport equation, in nondimensional form, is

$$
\frac{\partial \psi}{\partial t}+\mu \frac{\partial \psi}{\partial x}+\psi=\frac{c}{2} \int_{-1}^{1} \psi\left(x, \mu^{\prime}, t\right) d \mu^{\prime}
$$

We do not restrict the scattering ratio $c \leq 1$, but otherwise the notation is standard. We also specify a spatially uniform, isotropic initial condition,

$$
\psi(x, \mu, 0)=\frac{\Phi}{2},
$$

where $\Phi$ is the initial scalar flux, and vacuum boundary conditions,

$$
\psi(0, \mu, t)=0 \quad, \quad 0<\mu \leq 1,
$$

and

$$
\psi(L, \mu, t)=0 \quad, \quad-1 \leq \mu<0 \quad .
$$

Equations (2)-(4) were chosen for simplicity. However, we could extend what follows to more general initial and boundary conditions.

\section{ANALYTIC DISCRETE-ORDINATES SOLUTION}

The first step in solving Eqs. (1)-(4) via the ADO method is to approximate the angular dependence using quadrature. As is customary, we assume the quadrature set is symmetric about $\mu=0$ such that the directions are $\pm \mu_{n}\left(\mu_{n}>0\right)$ with weights $w_{n}, 1 \leq n \leq N$ (i.e., $2 N$ directions total), although we could adapt our approach to different types of quadrature. Equations (1)-(4) then become

$$
\begin{gathered}
\frac{\partial \psi_{n}^{ \pm}}{\partial t} \pm \mu_{n} \frac{\partial \psi_{n}^{ \pm}}{\partial x}+\psi_{n}^{ \pm}=\frac{c}{2} \sum_{m=1}^{N}\left(\psi_{m}^{+}+\psi_{m}^{-}\right) w_{m} \\
\psi_{n}^{ \pm}(x, 0)=\frac{\Phi}{2}
\end{gathered}
$$




$$
\psi_{n}^{+}(0, t)=0
$$

and

$$
\psi_{n}^{-}(L, t)=0
$$

where $\psi_{n}^{ \pm}(x, t)$ is the angular flux for direction $\pm \mu_{n}$. Next, we apply a Laplace transform in time to Eqs. (5)-(8), which yields

$$
\begin{gathered}
\pm \mu_{n} \frac{d \bar{\psi}_{n}^{ \pm}}{d x}+(s+1) \bar{\psi}_{n}^{ \pm}=\frac{c}{2} \sum_{m=1}^{N}\left(\bar{\psi}_{m}^{+}+\bar{\psi}_{m}^{-}\right) w_{m}+\frac{\Phi}{2} \\
\bar{\psi}_{n}^{+}(0, t)=0,
\end{gathered}
$$

and

$$
\bar{\psi}_{n}^{-}(L, t)=0
$$

Here, $\bar{\psi}_{n}^{ \pm}(x, s)$ is the Laplace transform of $\psi_{n}^{ \pm}(x, t)$ and $s$ is the transform parameter.

We look for homogeneous solutions to Eq. (9) separable in space and angle of the form

$$
\bar{\psi}_{n}^{ \pm}=\bar{\phi}_{n}^{ \pm} e^{-(s+1) x / \nu}
$$

By substituting this expression into Eq. (9) and setting $\Phi=0$, we can show that

$$
\bar{\phi}_{n}^{ \pm}=\frac{c}{2} \frac{1}{s+1} \frac{\nu}{\nu \mp \mu_{n}}
$$

up to a multiplicative constant, and

$$
\nu= \pm \sqrt{\xi}
$$

where $\xi$ is a root of

$$
F(\xi) \equiv 1-\frac{c}{s+1} \sum_{n=1}^{N} \frac{\xi}{\xi-\mu_{n}^{2}} w_{n}=0 .
$$

Equation (14) indicates there are two values of $\nu$, and hence two solutions of the form of Eq. (12), for each root of $F$. Also, an analysis of Eq. (15) reveals that (i) $F$ has $N$ roots total, which we denote $\xi_{k}, 1 \leq k \leq N$, and (ii) as $|s| \rightarrow \infty$,

$$
\xi_{k}=\mu_{k}^{2}+O\left(\frac{1}{s}\right)
$$

From the former, there are $2 N$ solutions of the form of Eq. (12), while the latter implies an ordering of the roots: the $k$ th root is the root that approaches $\mu_{k}^{2}$ as $|s| \rightarrow \infty$. Combining all the solutions of the form of Eq. (12) with a particular solution to Eq. (9) yields the general solution,

$$
\bar{\psi}_{n}^{ \pm}(x, s)=\sum_{k=1}^{N} A_{k} \bar{\phi}_{n, k}^{ \pm} e^{-(s+1) x / \nu_{k}}+\sum_{k=1}^{N} B_{k} \bar{\phi}_{n, k}^{\mp} e^{-(s+1)(L-x) / \nu_{k}}+\frac{\Phi}{2} \frac{1}{s+1-c} .
$$

In this expression,

$$
\bar{\phi}_{n, k}^{ \pm}=\frac{c}{2} \frac{1}{s+1} \frac{\nu_{k}}{\nu_{k} \mp \mu_{n}}
$$


and

$$
\nu_{k}=\sqrt{\xi_{k}},
$$

which follow from Eqs. (13) and (14), and $A_{k}$ and $B_{k}$ are as-yet unspecified coefficients [we have taken advantage of this to slightly rewrite the second sum in Eq. (17)].

To determine $A_{k}$ and $B_{k}$, we substitute Eq. (17) into Eqs. (10) and (11) and further use Eq. (18), which results in two $N \times N$ systems of equations,

$$
\mathbf{M}^{+} \mathbf{A}+\mathbf{M}^{-} \mathbf{D B}=-\frac{\Phi}{c} \frac{s+1}{s+1-c} \mathbf{1},
$$

and

$$
\mathbf{M}^{-} \mathbf{D A}+\mathbf{M}^{+} \mathbf{B}=-\frac{\Phi}{c} \frac{s+1}{s+1-c} \mathbf{1} .
$$

Here, $\mathbf{M}^{ \pm}$and $\mathbf{D}$ are $N \times N$ matrices given by

$$
\left(\mathbf{M}^{ \pm}\right)_{n, k}=\frac{\nu_{k}}{\nu_{k} \mp \mu_{n}},
$$

and

$$
\mathbf{D}=\operatorname{diag}\left[e^{-(s+1) L / \nu_{1}}, \ldots, e^{-(s+1) L / \nu_{N}}\right],
$$

$\mathbf{A}$ and $\mathbf{B}$ are $N \times 1$ vectors formed from the coefficients $A_{k}$ and $B_{k}$, respectively, and $\mathbf{1}$ is an $N \times 1$ vector consisting of ones. Instead of directly solving Eqs. (20) and (21) for A and B, however, we observe from these two expressions that $\mathbf{A}=\mathbf{B}$ and rewrite either as

$$
\left[\mathbf{I}+\left(\mathbf{M}^{+}\right)^{-1} \mathbf{M}^{-} \mathbf{D}\right] \mathbf{A}=-\frac{\Phi}{c} \frac{s+1}{s+1-c}\left(\mathbf{M}^{+}\right)^{-1} \mathbf{1}
$$

Then, because we will eventually need to consider $\operatorname{Re} s$ sufficiently large in order to invert the Laplace transform, we combine Eqs. (16) and (19) to show that as $|s| \rightarrow \infty$,

$$
\nu_{k}=\mu_{k}+O\left(\frac{1}{s}\right)
$$

and solve Eq. (24) in terms of a Neumann series,

$$
\mathbf{A}=-\frac{\Phi}{c} \frac{s+1}{s+1-c} \sum_{p=0}^{\infty}\left[-\left(\mathbf{M}^{+}\right)^{-1} \mathbf{M}^{-} \mathbf{D}\right]^{p}\left(\mathbf{M}^{+}\right)^{-1} \mathbf{1}
$$

such that, due to the exponential dependence in Eq. (23), the series converges if Re $s$ is large enough. For simplicity, we express Eq. (26) in the form

$$
\mathbf{A}=\frac{\Phi}{c} \frac{s+1}{s+1-c} \sum_{p=0}^{\infty} \mathbf{A}^{(p)}
$$

where $\mathbf{A}^{(p)}$ represents a sequence of $N \times 1$ vectors that satisfy

$$
\mathbf{M}^{+} \mathbf{A}^{(0)}=-\mathbf{1}
$$

and

$$
\mathbf{M}^{+} \mathbf{A}^{(p)}=-\mathbf{M}^{-} \mathbf{D} \mathbf{A}^{(p-1)} \quad, \quad p \geq 1 .
$$


We can solve these two expressions for $\mathbf{A}^{(p)}$ by employing a technique used by Chandrasekhar to solve similar systems of equations [1]. The resulting components of $\mathbf{A}^{(p)}, A_{k}^{(p)}$, are

$$
A_{k}^{(0)}=\frac{1}{\nu_{k}} \frac{\prod_{n=1}^{N} \mu_{n}-\nu_{k}}{\prod_{j=1, j \neq k}^{N} \nu_{j}-\nu_{k}}
$$

and

$$
A_{k}^{(p)}=\frac{1}{\nu_{k}} \frac{\prod_{n=1}^{N} \mu_{n}-\nu_{k}}{\prod_{j=1, j \neq k}^{N} \nu_{j}-\nu_{k}} \sum_{\ell=1}^{N} \frac{\nu_{\ell}}{\nu_{\ell}+\nu_{k}} \frac{\prod_{j=1}^{N} \nu_{j}+\nu_{\ell}}{\prod_{n=1}^{N} \mu_{n}+\nu_{\ell}} e^{-(s+1) L / \nu_{\ell}} A_{\ell}^{(p-1)}, \quad p \geq 1
$$

$A_{k}$ (and $B_{k}$ ) are thus given by Eqs. (27), (30), and (31).

Finally, we invert the Laplace transform by applying the usual Bromwich contour integral to Eq. (17), which results in

$$
\begin{aligned}
& \psi_{n}^{ \pm}(x, t)= \\
& \quad \frac{\Phi}{2} e^{(c-1) t}+\frac{1}{2 \pi i} \int_{\sigma-i \infty}^{\sigma+i \infty}\left[\sum_{k=1}^{N} A_{k} \bar{\phi}_{n, k}^{ \pm} e^{-(s+1) x / \nu_{k}}+\sum_{k=1}^{N} B_{k} \bar{\phi}_{n, k}^{\mp} e^{-(s+1)(L-x) / \nu_{k}}\right] e^{s t} d s .
\end{aligned}
$$

Here, $\sigma$ is a real constant chosen so that the contour is to the right of all the singularities of the integrand in the complex $s$ plane (i.e., Re $s$ is sufficiently large as mentioned above). We can show that the singularities consist of (i) a branch cut $-1<s<c-1$ and (ii) where $F$ has repeated roots, which can be determined from Eq. (15), as well.

\section{NUMERICAL EVALUATION}

Equation (32) is not amenable to numerical evaluation as is. In particular, the integrand is too oscillatory on the prescribed contour to allow numerical integration. We can deform the contour to something more easily integrated over, but first we need to better understand the behavior of the integrand in the complex $s$ plane. From examining Eqs. (30) and (31), we propose that $A_{k}^{(p)}$ has the form

$$
\begin{aligned}
& A_{k}^{(p)}= \\
& \frac{1}{\nu_{k}} \frac{\prod_{n=1}^{N} \mu_{n}-\nu_{k}}{\prod_{j=1, j \neq k}^{N} \nu_{j}-\nu_{k}} \sum_{q_{1}+\cdots+q_{N}=p} a_{k, q_{1}, \ldots, q_{N}} \prod_{j=1}^{N}\left[\frac{\prod_{n=1}^{N} \mu_{n}-\nu_{j}}{\prod_{\ell=1}^{N} \nu_{\ell}-\nu_{j} \prod_{n=1}^{N} \mu_{n}+\nu_{j}} e^{-(s+1) L / \nu_{j}}\right]^{q_{j}},
\end{aligned}
$$


for some coefficients $a_{k, q_{1}, \ldots, q_{N}}$. The sum in this expression is over all combinations of $N$ nonnegative integers that sum to $p$ (this is known as a weak composition). To determine the coefficients, we substitute Eq. (33) into Eqs. (30) and (31), which yields the following recursion relation

$$
a_{k, q_{1}, \ldots, q_{N}}=\sum_{j=1, q_{j} \geq 1}^{N} \frac{1}{\nu_{k}+\nu_{j}} a_{j, q_{1}, \ldots, q_{j}-1, \ldots, q_{N}} \quad, \quad q_{1}+\cdots+q_{N} \geq 1
$$

with initial value

$$
a_{k, 0, \ldots, 0}=1 .
$$

Equations (33)-(35) provide an alternative to Eqs. (30) and (31) for calculating $A_{k}^{(p)}$. Now, from Eqs. (25), (27), and (33), we see that the first sum in the integrand in Eq. (32) involves terms with exponential dependence that, as $\operatorname{Re} s \rightarrow \infty$, behave like

$$
e^{s t} e^{-(s+1) x / \nu_{k}} \prod_{j=1}^{N} e^{-(s+1) L q_{j} / \nu_{j}} \rightarrow O\left[e^{s\left(t-x / \mu_{k}\right)} \prod_{j=1}^{N} e^{-s L q_{j} / \mu_{j}}\right] .
$$

Therefore, if

$$
\frac{x}{\mu_{k}}+L \sum_{j=1}^{N} \frac{q_{j}}{\mu_{j}}>t
$$

we can deform the contour in Eq. (32) into the right complex $s$ plane to show that the contribution from that term can be neglected. Practically, we discard such terms by setting $a_{k, q_{1}, \ldots, q_{N}}=0$ in Eq. (33) [but not Eqs. (34) and (35)] when Eq. (37) is satisfied. Note that for $p=q_{1}+\cdots+q_{N}$ large enough, Eq. (37) is always satisfied so $A_{k}^{(p)}=0$, and consequently Eq. (27) reduces to a finite sum. Similarly, we consider the second sum in the integrand in Eq. (32) and obtain instead of Eq. (37)

$$
\frac{L-x}{\mu_{k}}+L \sum_{j=1}^{N} \frac{q_{j}}{\mu_{j}}>t
$$

We denote the coefficients that replace $A_{k}$ and $B_{k}$ in Eq. (32) when Eq. (33) is evaluated this way as $\tilde{A}_{k}$ and $\tilde{B}_{k}$, respectively.

From an analogous argument, the remaining terms in the integrand in Eq. (32) decay exponentially as $\operatorname{Re} s \rightarrow-\infty$ rather than oscillate. To take advantage of this behavior, we employ a method proposed by Talbot for integrals of this type [7]: we deform the contour in Eq. (32) such that it begins and ends in the left complex $s$ plane according to the parameterization

$$
s(\theta)=\alpha+\beta(i \gamma \theta+\theta \cot \theta) \quad, \quad-\pi<\theta<\pi .
$$

Here, $\alpha, \beta$, and $\gamma$ are constants chosen so that the contour remains to the right of the singularities discussed above. By substituting Eq. (39) and further using some properties of the integrand, we can write Eq. (32) as

$$
\begin{aligned}
& \psi_{n}^{ \pm}(x, t)=\frac{\Phi}{2} e^{(c-1) t} \\
&+\frac{1}{\pi} \int_{0}^{\pi} \operatorname{Im}\left\{\left[\sum_{k=1}^{N} \tilde{A}_{k} \bar{\phi}_{n, k}^{ \pm} e^{-(s+1) x / \nu_{k}}+\sum_{k=1}^{N} \tilde{B}_{k} \bar{\phi}_{n, k}^{\mp} e^{-(s+1)(L-x) / \nu_{k}}\right] e^{s t} \frac{d s}{d \theta}\right\} d \theta .
\end{aligned}
$$


This expression is readily amenable to numerical integration.

As an example, we have calculated the scalar flux for $L=2, c=1.5$, and $\Phi=1$ using uniform quadrature with $N=4$. In addition, we set $\alpha=\beta=2$ and $\gamma=1$. We found this choice of parameters sufficient for the calculations in this paper. The resulting contour and scalar flux at $t=1,2$, and 3 are shown in Fig. (1). Note the discontinuities in the right plot caused by ray effects.
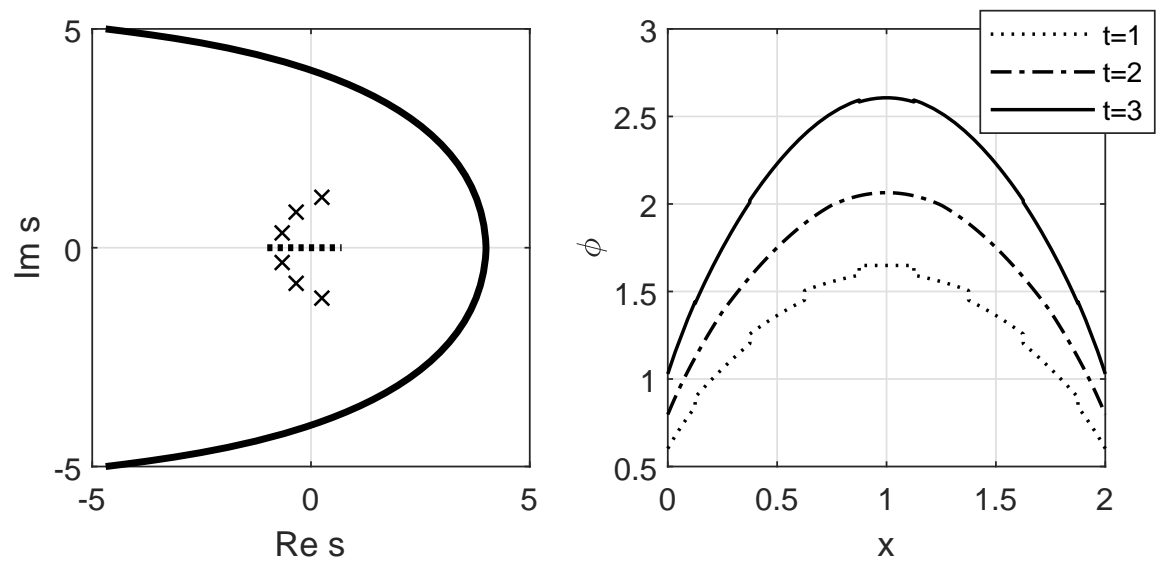

Figure 1: (Left) Contour used in example calculation. Repeated roots are denoted with an $\times$ and the branch cut with a dotted line. (Right) Scalar flux at different times.

\section{HIGH-PRECISION BENCHMARK}

For steady-state transport, the ADO method can be used to generate a high-precision benchmark by employing a sequence of quadrature sets with increasing $N$, then applying convergence acceleration to approximate the limit $N \rightarrow \infty$. For time-dependent transport, however, discontinuities caused by ray effects may interfere with convergence. We have found that by only using quadrature sets that yield exact results at the $x$ and $t$ coordinates of interest for $c=0$, convergence is sufficiently fast for $c>0$ even in the presence of such discontinuities. In most cases, this requirement is met by uniform quadrature with $N=a n, n=1,2,3, \ldots$, where $a$ is an integer "stride" that itself is a function of $x$ and $t$.

With this approach to selecting sequences of quadrature sets, we have calculated high-precision benchmark values of the scalar flux for the example problem described above $(L=2, c=1.5$, $\Phi=1$ ) at $x=1$ and various times. To apply convergence acceleration, for each $N$ we used the Wynn-epsilon algorithm [8] with the unaccelerated scalar-flux value and the six (or fewer for small $N)$ previous unaccelerated values in the sequence. The results are given in Table 1 to six figures, with the last digit being uncertain. The value of $N$ required to obtain the specified precision as well at the value of $a$ used for each time are also provided. 
Table 1: High-precision benchmark values of the scalar flux for the example problem at $x=1$ and various times. For each time, the value of $N$ required to obtain the specified precision as well as the value of $a$ used are also given.

\begin{tabular}{cccc}
\hline$t$ & $\phi$ & $N$ & $a$ \\
\hline 1.25 & 1.74718 & 50 & 5 \\
1.5 & 1.84382 & 60 & 3 \\
1.75 & 1.94745 & 49 & 7 \\
2.0 & 2.05970 & 40 & 2 \\
2.25 & 2.18102 & 63 & 9 \\
3.0 & 2.60113 & 54 & 3 \\
\hline
\end{tabular}

\section{CONCLUSIONS}

We have extended the ADO method to time-dependent transport in finite media. As with our previous work, the application of the ADO method to time-dependent transport is primarily accomplished through the use of a Laplace transform. The new aspect in this case is a system of equations that arises from enforcing boundary conditions. Instead of solving this system directly, we construct a solution in terms of Neumann series. We then discard terms when numerically evaluating the inverse Laplace transform such that the series reduces to a finite sum. We also use convergence acceleration with respect to the number of directions in the quadrature set to generate a high-precision benchmark.

\section{REFERENCES}

[1] S. Chandrasekhar, Radiative Transfer, Dover, Mineola, New York (1960).

[2] L.B. Barichello and C.E. Siewert, "Discrete-Ordinates Solution for a Non-Grey Model with Complete Frequency Redistribution," J. Quant. Spectrosc. Radiat. Transfer, 62, 665 (1999).

[3] C.E. Siewert, "A Concise and Accurate Solution to Chandrasekhar's Basic Problem in Radiative Transfer," J. Quant. Spectrosc. Radiat. Transfer, 64, 109 (2000).

[4] B.D. Ganapol, “The Response Matrix Discrete Ordinates Solution to the 1D Radiative Transfer Equation," J. Quant. Spectrosc. Radiat. Transfer, 154, 72 (2015).

[5] J.D. Densmore and G. Kooreman, "Analytic Discrete-Ordinates Solution for Time-Dependent Transport in Semi-Infinite Media," Trans. Am. Nucl. Soc., 121, 811 (2019).

[6] C.S. Aplin and D.L. Henderson, "Time-Dependent, One-Speed Integral Transport for OneDimensional Finite Slab Geometry," Trans. Am. Nucl. Soc., 99, 322 (2008).

[7] A. Talbot, "The Accurate Numerical Inversion of Laplace Transforms," J. Inst. Math. Appl., 23, 97 (1979).

[8] F. Bornemann, D. Laurie, S. Wagon, and J. Waldvogel, The SIAM 100-Digit Challenge, Society for Industrial and Applied Mathematics, Philadelphia, Pennsylvania (2004). 